\title{
Standardize the management procedures for breast cancer patients during the outbreak of COVID-19 in Wuhan, China
}

\author{
Jing Yao ${ }^{1}\left[\right.$ ? Yanfang Liu ${ }^{1} \cdot$ Jing Cheng ${ }^{1}$
}

Received: 22 March 2020 / Accepted: 11 June 2020 / Published online: 27 June 2020

○) Springer Science+Business Media, LLC, part of Springer Nature 2020

\begin{abstract}
Purpose The outbreak of the coronavirus disease 2019 (COVID-19) has led to interruption or delay in treatment of breast cancer patient. This commentary aims to standardize the management procedures and ensure complete or relatively complete treatment for breast cancer patients during the outbreak of COVID-19.

Methods Provide detailed online diagnosis, online treatment recommendations, and face-to-face consultation suggestions. Results Breast cancer patients who are at high risk of COVID-19 are advised to consult online first. For patients who have undergone online consultation and need face-to-face consultation, try to go to the clinic alone and take necessary precautions. Medical staff should be provided with necessary training about severe acute respiratory syndrome coronavirus 2 (SARSCoV-2) testing and knowledge of standard precautions and personal protective equipment.

Conclusions This commentary focused on breast cancer patients and provided suggestions to avoid the spread of COVID19. Some of these suggestions are also suitable for cancer patients in other lesions. We hope our suggestions are useful to oncologists in other countries and help them to overcome this challenge.
\end{abstract}

Keywords COVID-19 $\cdot$ SARS-CoV-2 $\cdot$ New coronavirus pneumonia $\cdot$ Breast cancer $\cdot$ Cancer therapy

The coronavirus disease 2019 (COVID-19) first started in Wuhan, China, and it soon appeared in other cities and overseas regions [1,2]. For the prevention and treatment of COVID-19, China has adopted many strict isolation measures, such as closing cities, restricting people activities, and blocking traffic. At present, the COVID-19 epidemic has been controlled in China; however, there are still many asymptomatic carriers. We as breast oncologists, are now focusing on the treatment of breast cancer patients. In order to ensure the diagnosis and treatment of breast cancer patients, it is necessary to carry out a reasonable and orderly consultation process.

Breast cancer patients who are at high risk of COVID19 are advised to consult online first, and then physicians recommend diagnosis and treatment strategies based on the

Jing Yao and Yanfang Liu have contributed equally to this work.

Jing Cheng

Chenjin1118@hotmail.com

1 Cancer Center, Union Hospital, Tongji Medical College, Huazhong University of Science and Technology, Jiefang Road 1277, Wuhan 430022, China patient's situation. The online diagnosis and treatment recommendations are as follows:

1. For routinely reviewed patients, it is recommended that the review be postponed without affecting the prognosis of the disease. For advanced breast cancer patients, the responsible physician should be contacted to evaluate the efficacy, go on further treatment, and make an appointment for the next examination 2 months in advance.

2. Promote online consultation to reduce hospital stay and avoid infection. Determining whether the patient can delay chemotherapy through online consultation. For advanced patients who are with low tumor load, old and weak, poor physical condition, and relatively slow tumor development, it's recommended to delay chemotherapy; oral endocrine therapy or oral chemotherapy or oral targeted drugs can be selected according to molecular typing. Try to minimize toxic and side effects on the premise of ensuring efficacy.

3. For early stage breast cancer patients who are undergoing adjuvant chemotherapy, it's recommended to delay chemotherapy. For locally advanced breast cancer 
patients who are undergoing adjuvant chemotherapy, try to schedule chemotherapy and combine targeted therapy according to the molecular type, such as HER-2 positive or triple negative breast cancer patients.

4. For advanced breast cancer patients with large tumor burden, rapid disease progression, and severe symptoms, it's recommended to make an appointment with doctor in advance to ensure that the treatment is performed normally if intravenous chemotherapy and targeted treatment are needed.

5. Patients who are undergoing adjuvant radiotherapy should not interrupt radiotherapy as far as possible. If radiotherapy has to be discontinued, the patients should record the last radiotherapy time. Supplement the dose appropriately according to the radiotherapy plan after the epidemic situation passes.

6. Patients who progress rapidly in the late stage and need radiotherapy to relieve symptoms, such as brain, bone, and soft tissue metastases, it's considered to implement large-segment radiotherapy to shorten the hospital stay if conditions permit.
7. For patients who plan to have breast cancer surgery, the surgery can be postponed if the tumor progresses slowly; for fast-developing malignancies, comprehensively evaluate the risk and perform the surgery under controlled conditions.

For patients who have undergone online consultation and need face-to-face consultation, the suggestions are as follows (Fig. 1a):

1. Try to go to the clinic alone. People with poor condition, minors under 18 years old, and patients over 70 years old are allowed to be accompanied by a family member. When patients and their family members go to the hospital, they are required to wear masks for protection. If you need to line up, it is recommended that the distance between people is more than $1.5 \mathrm{~m}$.

2. Patients and their families will receive temperature tests before entering the outpatient lobby. At the same time, the triage desk asked if patients are diagnosed with COVID-19 or suspected; whether they have fever, cough, and other respiratory tracts symptoms within
A

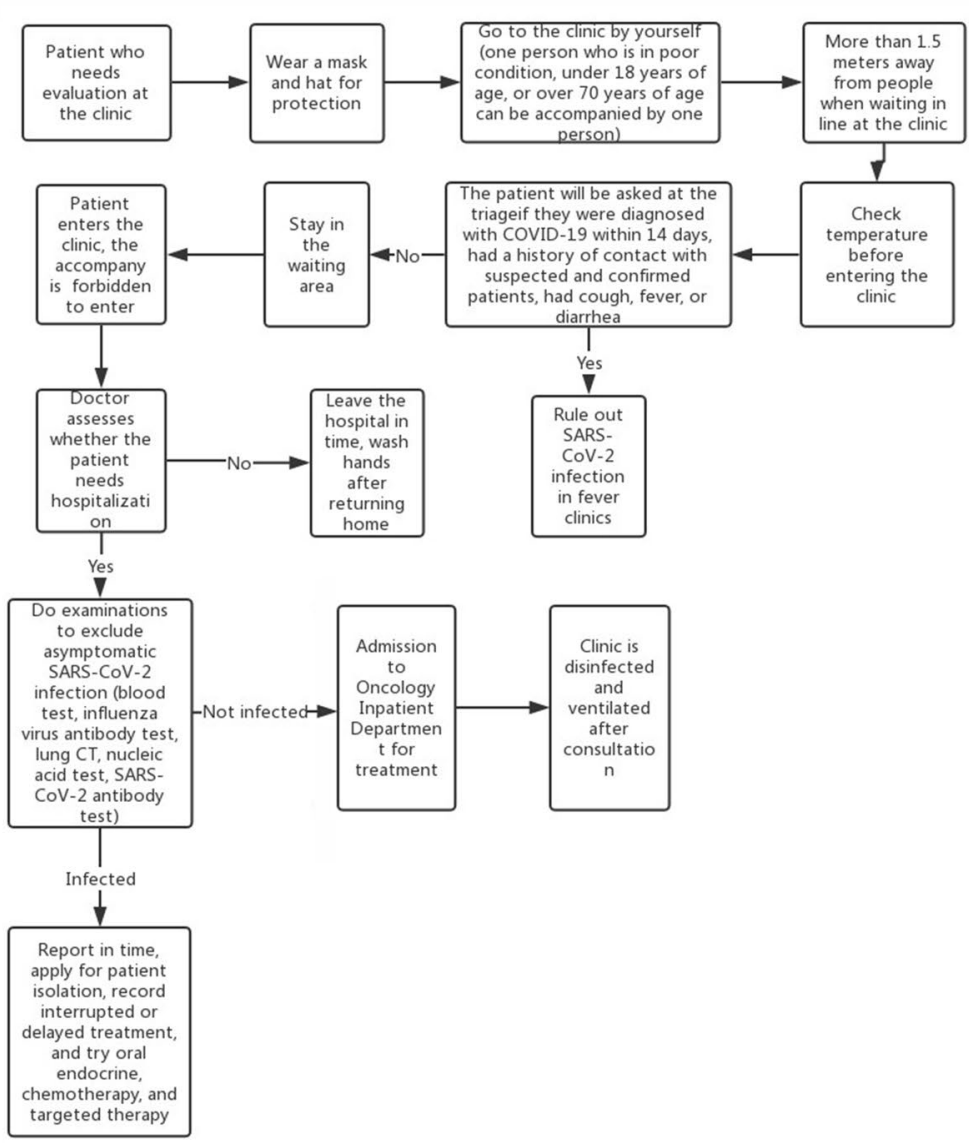

B

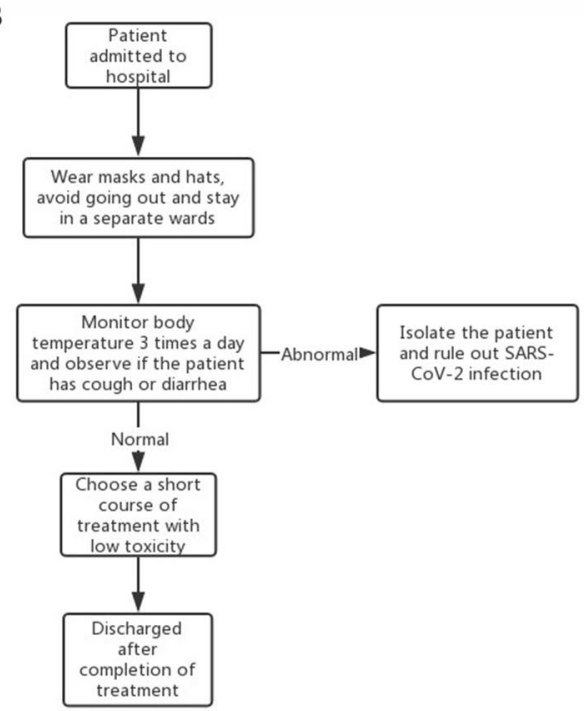

Fig. 1 Consultation process for breast cancer patients. a Outpatient consultation process. b Procedures for inpatients 
14 days before the visit; if they have a history of contacting with a suspected or confirmed COVID-19 patients, such as living, studying, working together, taking the same transportation; whether to participate in a dinner party with more than three people within 14 days before this visit. To speed up the consultation, above questions are recommended to provide as an option in a form. If the patient has fever and cough symptoms, it is recommended that the patient go to the fever clinics to rule out SARS-CoV-2 infection. The whole process is required to implement under good ventilation, and all the staff should wear masks, hats, and rubber gloves.

3. If the patient has no fever, cough, no related contact history, and no other symptoms of COVID-19, then he could be arranged for an oncology consultation.

4. The doctor should do three levels of protection. Only one patient is allowed in a clinic, and the patient cannot enter the clinic if the former patient has not finished the consultation. Family members are not allowed to accompany.

5. For patients who the doctor thinks need to be hospitalized, apply for nucleic acid detection, perform blood routine tests, influenza virus antibody tests, and lung CT. Family members also need to rule out infection. The doctors should report SARS-CoV-2 infections in time and apply for patient isolation.

6. The diagnosis room should be disinfected in time. It is recommended to use $1000 \mathrm{mg} / \mathrm{L}$ chlorine-containing disinfectant or hydrogen peroxide wet wipes for disinfection; 3\% hydrogen peroxide or $5000 \mathrm{mg} / \mathrm{L}$ peroxyacetic acid or $500 \mathrm{mg} / \mathrm{L}$ chlorine dioxide are used for air disinfection; UV lamp irradiation at least for $1 \mathrm{~h}$. Remember to open the window and ventilate after disinfection.

7. Urge breast cancer patients who can suspend or discontinue treatment to record details such as the interruption or delay of treatment. According to the stage and subtype, patients can be advised to switch to oral endocrine, chemotherapy, or targeted therapy.

Admission can be arranged for patients who are not infected with SARS-CoV-2 the suggestions are as follows (Fig. 1b):

1. After admission, if possible, arrange a separate ward for one patient as far as possible. Patients should wear masks during treatment and avoid going out. Visitors are not allowed unless necessary conditions.

2. During hospitalization, monitor the temperature of patients and the accompanied family three times a day, and observe whether they have symptoms such as cough and diarrhea. If necessary, apply for lung CT and nucleic acid test. If COVID-19 is considered, transfer and isolate the patient, disinfect the ward, and report it in time.
3. It is recommended to choose a short treatment course and low-toxicity chemotherapy to prevent patients from staying in the hospital for too long and increasing the risk of infection.

\section{Discussion}

During the outbreak of COVID-19, cancer patients are interrupted or delayed in review or treatment, which is not conducive to the patient's life and health. This commentary provides suggestions to ensure breast cancer patients' safety and treatment, and avoid cross-infection of COVID-19.

We also recommend that staff preparedness should not be ignored, such as providing clinic staff with necessary training about SARS-CoV-2 testing and use of standard precautions and personal protective equipment, which are also important measures to prevent SARS-CoV-2 infection according to American Society of Clinical Oncology (ASCO) guidance [3]. Nowadays, as more and more asymptomatic carriers are diagnosed [4], we strongly recommend that SARS-CoV-2 testing and lung CT be listed as routine inspection items for every patient. After the outbreak of COVID-19 in Wuhan, China, medical teams from other provinces came to Wuhan to participate in the rescue. When they finish the rescue and return to their work places, they will be quarantined for 14 days before starting normal work.

This commentary focused on breast cancer patients and provided suggestions to avoid the spread of COVID19. Some of these suggestions are also suitable for cancer patients in other lesions. We hope our suggestions are useful to oncologists in other countries and help them to overcome this challenge.

Funding No support from any organization for the submitted work.

\section{Compliance with ethical standards}

Conflict of interest All the authors declared no conflict of interests.

Ethical approval This article does not contain any studies with human participants or animals performed by any of the authors.

\section{Reference}

1. Kim JY, Choe PG, Oh Y et al (2020) The first case of 2019 novel coronavirus pneumonia imported into Korea from Wuhan, China: implication for infection prevention and control measures. J Korean Med Sci 35(5):e61. https://doi.org/10.3346/jkms.2020.35. e61

2. Tang B, Wang X, Li Q et al (2020) Estimation of the transmission Risk of the 2019-nCoV and its implication for public health interventions. J Clin Med. https://doi.org/10.3390/jcm9020462 
3. Oncology ASOC (2020) COVID-19 provider \& practice information. https://www.asco.org/asco-coronavirus-information/provi der-practice-preparedness-covid-19. Accessed 9 June 2020

4. Lai CC, Liu YH, Wang CY et al (2020) Asymptomatic carrier state, acute respiratory disease, and pneumonia due to severe acute respiratory syndrome coronavirus 2 (SARS-CoV-2): facts and myths. J Microbiol, Immunol, Infect. https://doi.org/10.1016/j. jmii.2020.02.012
Publisher's Note Springer Nature remains neutral with regard to jurisdictional claims in published maps and institutional affiliations. 